\title{
Sobre el producto cruz en espacios vectoriales n-dimensionales
}

\author{
M.A. Murray-Lasso \\ Unidad de Enseñanza Auxiliada por Computadora \\ Departamento de Ingeniería de Sistemas. División de Estudios de Posgrado \\ Facultad de Ingeniería, UNAM \\ E-mail:mamurray@servidor.unam.mx
}

(recibido: junio de 2002; aceptado: mayo de 2003)

\section{Resumen}

A diferencia del producto interno entre vectores que se define sin problemas como una operación binaria para vectores en $n$ dimensiones, el producto vecto rial o producto cruz normalmente se define solamente para vectores tridimensionales. Si en vez de insistir en que el producto vecto rial es una operación binaria, se reconoce que resulta más benéfico considerarla como una operación $(n-1)$-aria, donde $n$ es la dimensión del espacio, (en tres dimensiones como $n-1=2$, no hay diferencia entre binaria y $(n-1)$-aria). La generalización del producto vecto rial a cualquier número de dimensiones a partir de $n=1$, procede fácilmente y con aplicaciones útiles. En este artículo se define un producto vectorial entre $n-1$ vectores $n$-dimensionales. Se dan dos aplicaciones con ejemplos ilustrativos de dicha definición: al cálculo de volúmenes de paralelepípedos y simplejos n-dimensionales que son las generalizaciones a $n$ dimensiones del paralelogramo y paralelelpípedo y del triángulo y tetraedro, así como a la solución de ecuaciones lineales simultáneas. Los desarrollos se basan en las propiedades de los determinantes.

Descriptores: producto vectorial, producto cruz, n-dimensional, hiperárea, hipervolumen, simplejo.

\begin{abstract}
Con trasting with the in nerprod uct be tween vectors which is de fined with out prob lems as a bi nary op er a tion in n di men sions, the vec tor or cross prod uct is nor mally de fined only for three-dimensional vec tors. If in stead of in sist ing that the cross prod uct is a bi nary op er a tion it is recog nized that it is more use ful to con sider it as an (n-1)-ary op er a tion, where $n$ is the di men sion of the space, (in three di men sions, since $n-1=2$, there is no dif fer ence be tween bi nary and ( $n-1)$-ary op er a tions.) With this pro vi sion, the gen er al iza tion of the cross prod uct to any num ber of di men sions from one on, pro ceeds very nat $u$ rally and has use ful ap plica tions. In this ar ti cle we de fine a cross prod uct be tween $n-1 n$-dimensional vectors. Two ap plications are given with il lus trative examples to the calcu lation of volumes of n-dimensional par al lel epi peds and simplices, which are the gen er al iza tions to ndimensions of the par al lel o gram and parallelepiped and the tri an gle and tet ra he dron and to the solution of sets ofsimultaneous linearequations. The de velopments are based of the properties of de terminants.
\end{abstract}

Keywords: vec tor prod uct, cross-product, $n$-dimensional, hyperarea, hypervolume, simplex. 


\section{Introducción}

La geometría plana y tridimensional euclideana se ha generalizado para admitir versiones en $n$ dimensiones. Dado que nuestra intuición reconoce solamente 3 dimensiones, para manejar la geometría $n$-dimesional es necesario hacerlo analíticamente. La principal herramienta para realizarlo es la teoría de vectores, aunque también juegan un papel importante la geometría analítica en $n$ dimensiones y el álgebra lineal (Aleksandrov et al., 1963 y Smirnov, 1970). Existe una rica y útil teoría de vectores en $n$ dimensiones que es una extensión por analogía de conceptos tridimensionales. A continuación, con el propósito de comenzar a tratar el tema sin tener que introducir con toda precisión y completez la teoría de vectores $\mathrm{n}$-dimensionales, se esboza de una manera informal y simplificada los principales conceptos, refiriendo al lector a algún libro como (Birkhoff y Mac Lane 1965) para detalles y tratamientos más rigurosos.

Aunque no se pueda representar en términos geométricos convencionales para $n>3$, se postula como sistema de referencia un conjunto de $n$ ejes ortogonales entre sí que parten desde un punto especial llamado origen, al cual denotaremos con $\mathrm{O} y$ postulamos $n$ vectores unitarios (con longitud 1) orientados en la dirección positiva de cada uno de los ejes. A estos vectores unitarios les llamaremos $\boldsymbol{i}, \boldsymbol{j}, \boldsymbol{k}, \ldots, \boldsymbol{l}$. Elegido un sistema de referencia (origen y $n$ ejes orientados ortogonales entre sí con sus $n$ vectores unitarios), cualquier punto $X$ del espacio queda determinado por $n$ coordenadas $\left(x_{1}\right.$, $\left.x_{2}, \ldots, x_{n}\right)$ cuyo significado es que el punto $X$ corresponde con la punta de un vector n-dimesional $\boldsymbol{X}$ que arranca del origen y queda expresado de una manera única por la siguiente expresión

$$
\boldsymbol{X}=x_{1} \boldsymbol{i}+x_{2} \boldsymbol{j}+x_{3} \boldsymbol{k}+\ldots+x_{n} \boldsymbol{l}
$$

La suma de vectores $\mathrm{n}$-dimensionales es una extensión de la fa mil iar Ley del Polígono, en la cual se colocan los sumandos desplazando las flechas paralelas a sí mismas (sin cambiar dirección o sentido), de tal manera que el primer sumando apoya la cola del vector en el origen, el segundo sumando apoya su cola en la punta del primer sumando, ... , el iésimo sumando apoya su cola en la punta del (i-1)-ésimo sumando, ..., el n-ésimo sumando apoya su cola en la punta del (n-1)-ésimo sumando. El resultado de la suma es el vec tor que va del origen a la punta del $n$-ésimo sumando. Para tres dimensiones, la figura 1 ilustra el proceso. Para $n$ dimensiones $(n>3)$ a falta de una interpretación geométrica se define la suma $\boldsymbol{u}+\boldsymbol{v}$ de los vectores $\boldsymbol{u}=\left(u_{1}, u_{2}, \ldots, u_{n}\right)$ y $\boldsymbol{v}$ $=\left(v_{1}, v_{2}, \ldots, v_{n}\right)$ de la siguiente manera (la definición es válida también para vectores en espacios con $n \leq 3$ dimensiones)

$$
\boldsymbol{u}+\boldsymbol{v}=\left(u_{1}+v_{1}, u_{2}+v_{2}, \ldots, u_{n}+v_{n}\right)
$$

Esta definición coincide con la interpretación geométrica para $n \leq 3$.

Para que el proceso quede bien definido es necesario decir que se entiende por el producto de un escalar como $x_{1}$ por un vector como $\boldsymbol{i}$ en la expresión de $\boldsymbol{X}$. (Aunque en tratamientos más completos el conjunto de escalares puede ser cualquier campo, aquí, solamente consideraremos escalares que son números reales). Si el escalar es positivo, el resultado es otro vector con la 


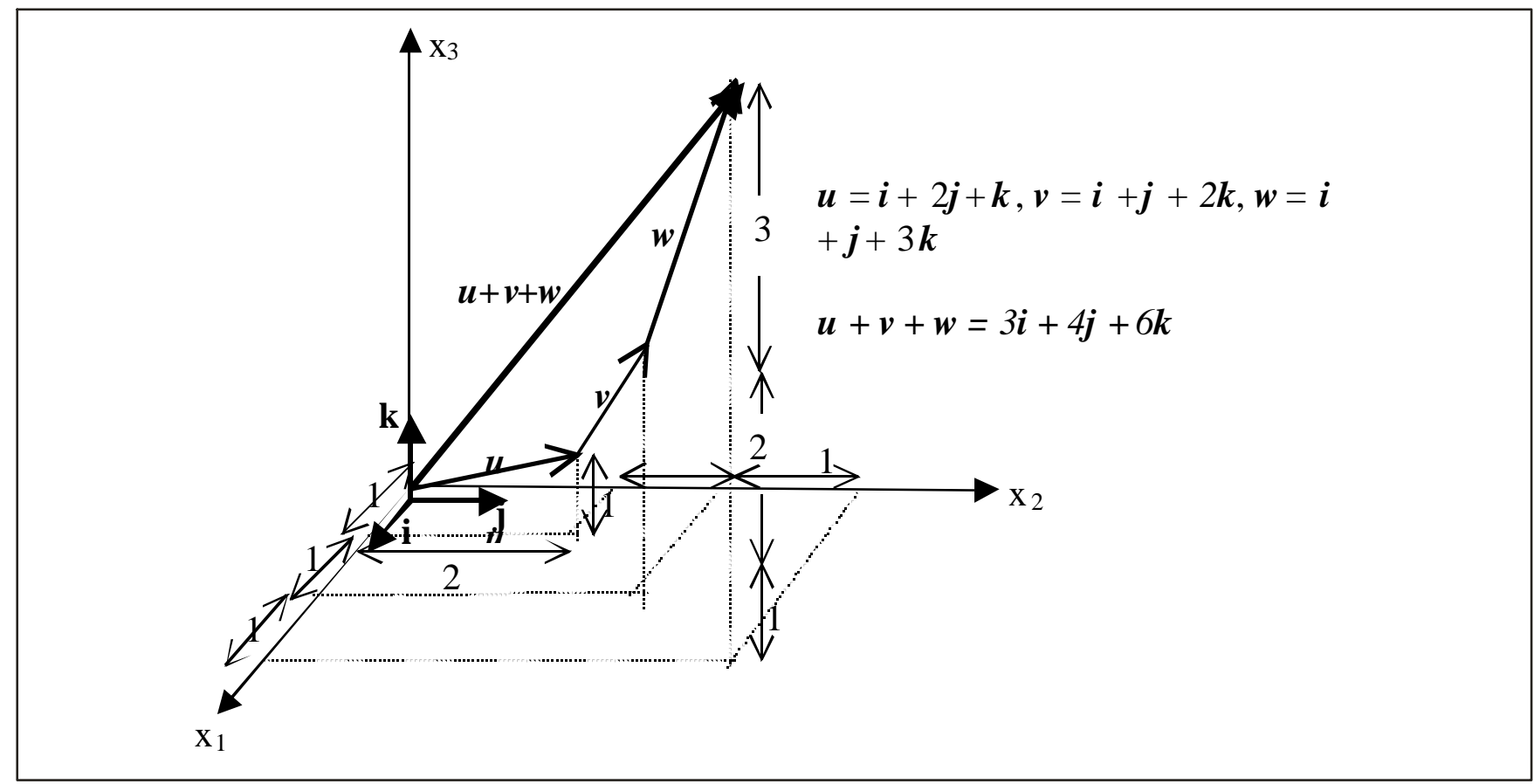

Figura 1

misma orientación y sentido que el vector orig i nal, pero con una longitud $x_{1}$ veces más larga (o más corta si el número es menor que 1). Si el escalar es negativo el resultado es el mismo pero con el sentido invertido. Cuando el escalar es cero el producto da como resultado el vec tor cero representado con $\mathbf{0}$, que es un vector con cero longitud y se representa con un punto en el origen, además de tener la propiedad de que al sumarle el vec tor cero a cualquier otro vec tor el resultado es el mismo vector.

Analíticamente podemos definir la multiplicación del escalar a por el vector $\boldsymbol{X}=\left(x_{1}\right.$, $\left.x_{2}, \ldots, x_{n}\right)$ como el vec tor $a \boldsymbol{X}=\left(a x_{1}, a x_{2}, \ldots\right.$ . , $a x_{n}$ ). El producto de un escalar por un vector está cerrado en el espacio vec to rial y por definición es lo mismo av que va, es decir, el producto de un escalar por un vector es conmutativo por definición. También es necesario decir qué se entiende por la longitud de un vector, ya que hemos hablado de vectores unitarios y de cómo en el producto entre un escalar positivo y un vector, el vec tor se alarga o se acorta.

La longitud o magnitud de un vec tor $\boldsymbol{X}$ que normalmente se denota con $|\boldsymbol{X}|$ es por definición,

$$
|\boldsymbol{X}|=\sqrt{x_{1}^{2}+x_{2}^{2}+\ldots+x_{n}^{2}}
$$

En la expresión anterior se toma el valor positivo de la raíz cuadrada.

Aunque ya se mencionó que los ejes son ortogonales entre sí, y por lo tanto los vectores unitarios también, completamos la definición de ortogonalidad definiendo el producto interno entre dos vectores $\boldsymbol{x}=\left(x_{1}\right.$, $\left.x_{2}, \ldots, x_{n}\right)$ y $\boldsymbol{y}=\left(y_{1}, y_{2}, \ldots, y_{n}\right)$. El producto interno entre dos vectores $\boldsymbol{x}, \boldsymbol{y}$ es un número (técnicamente se llama un escalar) que representamos con $\boldsymbol{x}$.y cuyo valor está dado por

$$
\boldsymbol{x} \cdot \boldsymbol{y}=x_{1} y_{1}+x_{2} y_{2}+\ldots+x_{n} y_{n}
$$


Cuando el producto interno entre dos vectores vale cero, decimos que los vectores son ortogonales. Por lo tanto, debido a que los vectores unitarios están sobre ejes ortogonales entre sí y ordenados $\mathbf{i}, \mathbf{j}, \mathbf{k}, \ldots, \mathbf{I}$ se tiene $\mathbf{i}=(1,0,0, \ldots, 0), \mathbf{j}=(0,1,0, \ldots, 0)$, $\ldots, \mathbf{I}=(0,0, \ldots, 1)$ por lo que

$$
\begin{gathered}
i \cdot i=j \cdot j=\ldots=l . l=l ; \quad i \cdot j=j \cdot i=i \cdot k=k \cdot i= \\
\ldots=h . l=l . h=0
\end{gathered}
$$

es decir, el producto interno (también llamado producto punto o producto escalar) entre cualquier par de vectores unitarios diferentes de un sistema de ejes ortogonales es el escalar cero, mientras que el producto escalar consigo mismo es la unidad.

La operación de suma de dos vectores da como resultado un vec tor y está cerrada en el espacio vectorial (es decir, cualquier par de vectores se pueden sumar sin salirse del espacio vec to rial). La suma de vectores tiene varias de las mismas propiedades que la suma de números entre las que están la conmutativa y asociativa. Con respecto a la multiplicación entre escalares y vectores, la suma de vectores cumple la ley distributiva y la suma de escalares también distribuye con respecto a los vectores, es decir,

$$
\begin{aligned}
& a(\boldsymbol{u}+\boldsymbol{v})=a \boldsymbol{u}+a \boldsymbol{v} \\
& (a+b) \boldsymbol{u}=a \boldsymbol{u}+b \boldsymbol{u}
\end{aligned}
$$

Para el producto escalar y la suma vectorial también se cumple la ley distibutiva, es decir,

$$
\mathbf{u} \cdot(\mathbf{v}+\mathbf{w})=\mathbf{u} \cdot \mathbf{v}+\mathbf{u} \cdot \mathbf{w}
$$

La longitud de un vector $\boldsymbol{v}$ también se puede expresar por la fórmula

$$
|v|=(v \cdot v)^{1 / 2}
$$

y la distancia entre los puntos $P=\left(p_{1}, p_{2}, \ldots\right.$, $\left.p_{n}\right)$ y $Q=\left(q_{1}, q_{2}, \ldots, q_{n}\right)$ está dada (como postulado) por

$d(P, Q)=\left[\left(p_{1}-q_{1}\right)^{2}+\left(p_{2}-q_{2}\right)^{2}+\ldots+\left(p_{n}-q_{n}\right)^{2}\right]^{1 / 2}$

Reconocemos en la última expresión una generalización a $n$ dimensiones del Teorema de Pitágoras para triángulos rectángulos. En la fórmula anterior siempre se toma la raíz cuadrada positiva. También se puede definir el coseno del ángulo entre dos vectores en un espacio $n$-dimesional como sigue:

$$
\cos \theta=\mathbf{u . v} /|u| v
$$

\section{Apoyo en la intuición de espacios n-dimensionales}

Aunque nuestra experiencia e intuición corresponden a tres dimensiones, podemos ayudarla en más dimensiones, considerando por ejemplo, la cuarta dimensión (Davis y Hersh, 1981). Una línea recta se forma desplazando (y pintando el espacio al desplazarse) un punto en cualquier dirección. Un paralelogramo se forma desplazando una línea (y pintando con la línea el espacio al desplazarse) en una dirección diferente de la dirección de la línea. Un paralelepípedo se forma desplazando un paralelogramo en una dirección diferente al plano en el que está y pintando el espacio al desplazarse. Un paralelepípedo en cuatro dimensiones se forma desplazando un paralelepípedo en tres dimensiones en una dirección diferente a las tres en las que está el paralelepípedo que se desplaza. Imaginándonos lo anterior, los 8 vértices del paralelepípedo en tres dimensiones, generan 8 aristas adicionales a las que 
tienen los paralelepípedos en sus posiciones inicial (antes de iniciar el desplazamiento) y final (al terminar el desplazamiento). De acuerdo con esto, un paralelepípedo en cuatro dimensiones tendrá $12 \times 2+8=32$ aristas. Tendrá $8 \times 2=16$ vértices. Sus caras tridimensionales (que son los paralelepípedos tridimensionales generados por las caras bidimensionales del paralelepípedo en tres dimensiones al desplazarse en una dirección que no está en las tres dimensiones del cuerpo deplazado). Cada cara bidimensional genera una nueva cara tri di men sion al a las que se les añaden el paralelepípedo inicial y final. Por lo tanto, el paralelepípedo en cuatro dimensiones tiene $6+2=8$ caras tridimensionales. Cada arista genera con el movimiento una cara bidimensional (un paralelogramo) más las caras de los paralelepípedos inicial y final, por lo tanto, el cuerpo tetradimensional tiene 12+ $12=24$ caras bidimensionales. (Las aristas mencionadas arriba, serían caras unidimensionales y los vértices caras cerodimensionales). No obstante, nuestra limitación a tres dimensiones, se pudo deducir varias cosas de un cuerpo tetradimensional. Para mejor visualización, en vez de paralelogramos se puede pensar en cuadrados y contar elementos de un cubo tetradimensional. La figura $2 a$ muestra un cubo tetradimensional proyectado sobre tres dimensiones y posteriormente dibujado por medio de una proyección sobre un plano bidimensional. La figura $2 \mathrm{~b}$ muestra un simplejo tetradimensional en iguales circunstancias.

Otro cuerpo tetradimensional que nos interesa estudiar es el simplejo tetradimensional. Este cuerpo es la generalización del segmento de recta, triángulo y tetraedro. Comenzamos con un punto para formar un simplejo cero dimensional. A continuación introducimos un segundo punto diferente del primero. El segmento de recta que une los dos puntos, constituye un simplejo unidimensional. A continuación escogemos un tercer punto que no esté en línea recta con los primeros dos puntos. Se forma un
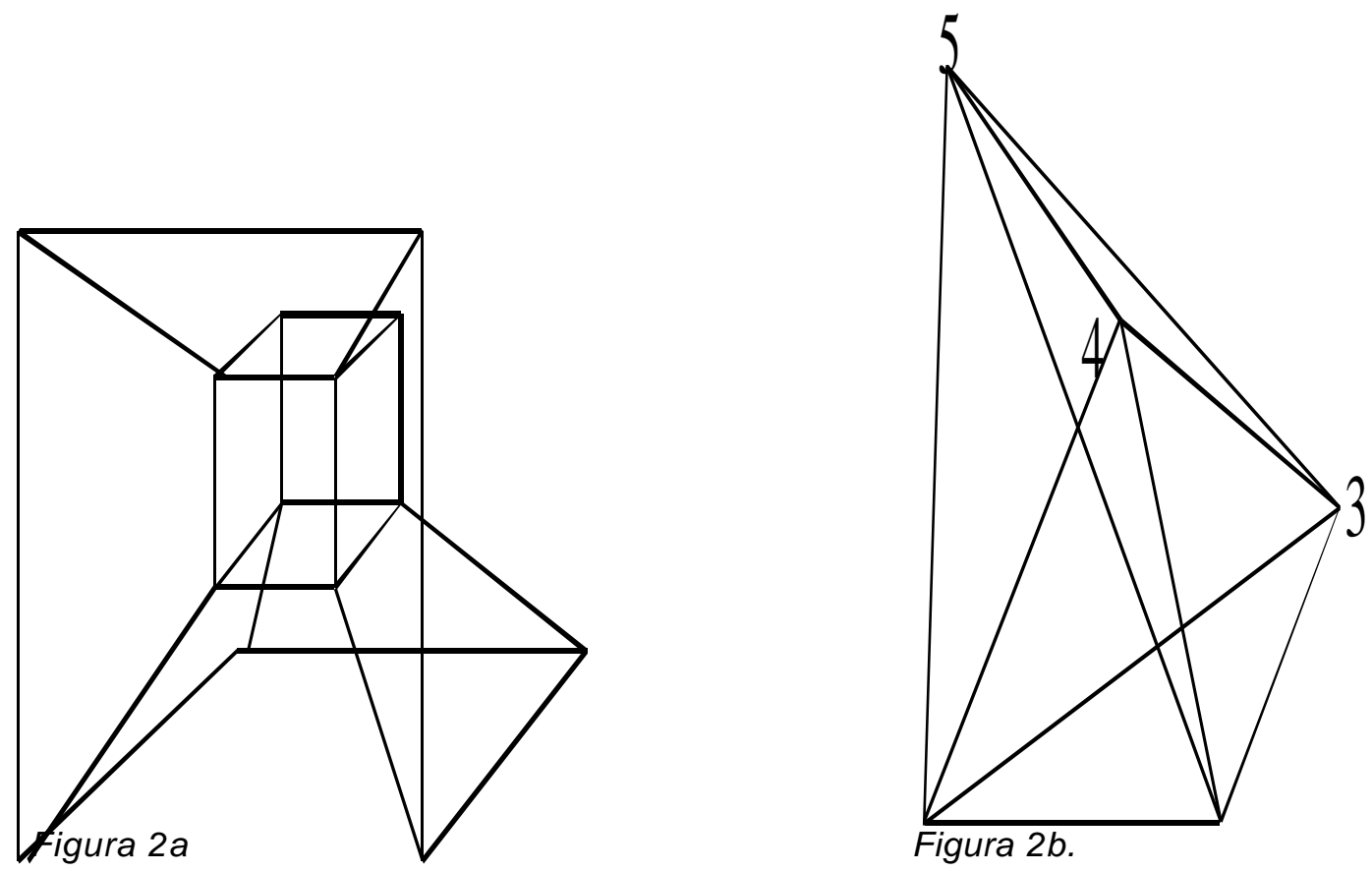
triángulo que es el conjunto de todos los puntos en las líneas rectas que unen los puntos del simplejo unidimensional con el nuevo punto. A continuación escogemos un cuarto punto que no esté en el mismo plano que el triángulo. El tetraedro es el conjunto de todos los puntos de las rectas que unen el nuevo punto con todos los puntos del triángulo. Finalmente, escogemos un quinto punto que no esté en el espacio tri di men sional en el que está el tetraedro. El simplejo tetradimensional es el conjunto de puntos en las rectas que unen el nuevo punto con todos los puntos del tetraedro. Siguiendo el proceso se definen simplejos de más dimensiones Dubrovin et al (1987) ¿Cuántos vértices, aristas y caras tienen un tetraedro y un simplejo tetradimensional? Bueno, en cuanto a vértices siempre tienen uno más que el número de dimensiones. Por lo tanto, el tetraedro tiene 4 vértices y el simplejo tetradimensional tiene 5. En cuanto a aristas, el tetraedro tiene 6 aristas, 3 del triángulo base y una más por cada una de las líneas que van del nuevo punto a cada uno de los vértices del triángulo base. El simplejo tetradimensional tiene 6 aristas del tetraedro base y 4 más que van del nuevo punto a cada uno de los 4 vértices del tetraedro. En cuanto a caras bidimensionales, el tetraedro tiene 4 caras (de ahí su nombre). El simplejo tetradimensional tiene las mismas del tetraedro más una cara por cada arista del tetraedro base que al unir sus dos extremos con el quinto punto forma un triángulo adicional, por lo que el simplejo tetradimensional tiene 10 caras triangulares (bidimensionales).

También el simplejo tetradimensional tiene caras tridimensionales que son tetraedros; comenzamos por el tetraedro base y luego sobre cada una de 4 las caras triangulares de este tetraedro base se apoya otro tetraedro que tiene como cúspide el quinto punto del simplejo. Entonces existen 5 caras tetraédricas de tres dimensiones que "envuelven" al simplejo tetradimensional. Lo anterior se ilustra en la figura $2 b$. Las figuras $2 a$ y $2 b$ son del tipo de alambre, porque se pretende dibujar solamente las aristas sin intentar hacer opacas las caras bidimensional que taparían unas a otras. En cuerpos como los simplejos, en los cuales se conectan todos los vértices, la cuenta del número de "caras" de diferentes dimensiones se puede lograr usando cálculo combinatórico. Se comienza con el número de vértices que es $n+1$ (uno más que el número de dimensiones $n$ ). El número de aristas es entonces el número de combinaciones de $n+1$ objetos tomados de 2 en dos. Es decir,

$C_{2}^{n+1}=\left(\begin{array}{c}n+1 \\ 2\end{array}\right)=\frac{(n+1) !}{(n-2) ! 2 !}=\frac{5.4}{1.2}=10 \quad($ utilizando $n=4)$

El número de caras triangulares es el número de combinaciones de $n+1$ objetos tomados de 3 en 3.

$$
C_{3}^{n+1}=\frac{5.4 .3}{1.2 .3}=\frac{60}{6}=10
$$

El número de caras tetraedrales es el número de combinaciones de $n+1$ objetos tomados de 4 en 4.

$$
C_{4}^{n+1}=\frac{5 \cdot 4 \cdot 3 \cdot 2}{1 \cdot 2 \cdot 3 \cdot 4}=\frac{120}{24}=5
$$

En este artículo también interesa calcular el volumen de paralelepípedos y simplejos de diversos números de dimensiones. El cálculo 
del volumen de un paralelepípedo, comenzando con un paralelogramo que es la instancia de este cuerpo en dos dimensiones, se hace calculando la medida de la base (en caso de que la base sea una línea recta, su longitud; si es bidimensional, su área; si tridimensional, su volumen, y en casos de mayor dimensión, su hipervolumen) y multiplicando la medida de la base por la distancia entre la base y la "cara" paralela a la misma. La "cara" para un paralelogramo, es un segmento de recta; para una base plana, un paralelogramo; para una base tridimensional un paralelepípedo; etc. En el caso de un simplejo también se calcula la medida de la base y se multiplica por la altura (distancia entre la cúspide y la base) y adicionalmente se divide entre el número de dimensiones (para un triángulo se di vide entre 2 , para un tetraedro se divide entre 3; para un simplejo de 4 dimensiones se divide entre 4, etc). Si se acumulan los factores entre los que hay que dividir, el volumen de un paralelepípedo $n$-dimensional es $n$ ! veces mayor que el volumen de un simplejo de igual dimensión generado por los mismos $n+1$ vectores $n$-dimensionales. Nótese que el proceso es recursivo, pues para calcular la medida de la base de un paralelpípedo o de un simplejo $n$-dimensional se puede utilizar la expresión para el cálculo de la medida de un cuerpo (n-1)-di men sional.

\section{El producto cruz o producto vectorial en tres dimensiones}

Para tres dimensiones el producto vectorialo producto cruz $\boldsymbol{u} \times \boldsymbol{V}$ de los vectores $\boldsymbol{u}=\left(u_{1}\right.$, $\left.u_{2}, u_{3}\right)$ y $\boldsymbol{v}=\left(v_{1}, v_{2}, v_{3}\right)$ se de fine de la siguiente manera (Thomas, 1960):

"Sea $\theta$ el ángulo entre los vectores $\boldsymbol{u}$ y $\boldsymbol{v}$ no colineales, donde $0 \leq \theta \leq \pi$, los vectores $\boldsymbol{u}$ y $\boldsymbol{v}$ determinan un plano cuando ambos parten del origen; sea $\boldsymbol{n}$ un vector unitario perpendicularal plano de $\boldsymbol{u}$ y $\boldsymbol{v}$ y apuntando en el sentido en el que avanzaría un tornillo de rosca derecha cuando se gira un ángulo $\theta \leq \pi$ que sobreponga el vector $\boldsymbol{u}$ sobre el vector $\boldsymbol{v}$, entonces el producto cruz o producto vec to rial $\boldsymbol{u} \times \boldsymbol{v}$ está dado por

$$
\boldsymbol{u} \times \boldsymbol{v}=\boldsymbol{n} \boldsymbol{u} \| \boldsymbol{v} \mid \operatorname{sen} \theta
$$

Si se utilizan las componentes cartesianas de $\boldsymbol{u}$ y $\boldsymbol{v}$ se puede demostrar que formalmente

$$
\begin{gathered}
\boldsymbol{u} \times \boldsymbol{v}=\left|\begin{array}{ccc}
\boldsymbol{i} & \boldsymbol{j} & \boldsymbol{k} \\
u_{1} & u_{2} & u_{3} \\
v_{1} & v_{2} & v_{3}
\end{array}\right| \begin{array}{lll}
u_{1} & u_{2} & u_{3} \\
v_{1} & v_{2} & v_{3} \\
\boldsymbol{i} & \boldsymbol{j} & \boldsymbol{k}
\end{array} \mid= \\
\left(u_{2} v_{3}-v_{2} u_{3}\right) \boldsymbol{i}-\left(u_{1} v_{3}-v_{1} u_{3}\right) \boldsymbol{j}+\left(u_{1} v_{2}-v_{1} u_{2}\right) \boldsymbol{k}
\end{gathered}
$$

donde $\boldsymbol{i}, \boldsymbol{j}, \boldsymbol{k}$, son los vectores unitarios en la dirección de los 3 ejes cartesianos en un sistema derecho, es decir, que los ejes están orientados de manera que para una mano derecha el pulgar se puede hacer coincidir con $\boldsymbol{i}$, el índice con $\boldsymbol{j}$ y el anular con $\boldsymbol{k}$, (Thomas, 1960). De los dos determinantes en la última ecuación vamos a preferir el segundo, debido a que para tres dimensiones ambos son equivalentes, para un número par de dimensiones nos ahorraremos un factor igual a - 1 elevado a una potencia que cambia con el número de dimensiones. La definición con el segundo determinante asegura que para cualquier $n$, el producto cruz de los primeros $n-1$ vectores unitarios produce el último vec tor unitario, por lo tanto, el juego de vectores unitarios tiene una orientación "derecha." El producto vectorial en tres dimensiones no es conmutativo (de 
hecho es anticonmutativo) pues cuando se invierte el orden de los factores se invierte el sentido del vector resultante, pues en el deter- minante se intercambian dos filas. También resulta que si los dos vectores $\boldsymbol{u}$ y $\boldsymbol{v}$ son colineales (y por lo tanto proporcionales en sus componentes) su producto cruz es el vector cero. Una interpretación geométrica del producto cruz entre dos vectores tridimensionales es que la magnitud del vector resultante es igual al área del paralelogramo determinado por los dos vectores como se ilustra en la figura 3. Por las propiedades de los determinantes, el producto cruz obedece la Ley Distributiva con respecto a la suma (tanto vec to rial como escalar).

Son muy pocos los libros sobre vectores n-dimensionales que mencionan el producto cruz (Lang, 1966 y Xambó, 1997) y los que lo estudian se limitan a tratar espacios de tres dimensiones (Hague, 1951 y Hoffmann, 1966).

\section{Productos mixtos y volúmenes de paralelepípedos en tres y más dimensiones}

En vista de que el producto cruz $\boldsymbol{u} \times \boldsymbol{v}$ de dos vectores $\boldsymbol{u}, \boldsymbol{v}$ en tres dimensiones es un vector tridimensional, se puede concebir hacer un producto escalar o producto punto del producto vec to rial $\boldsymbol{u} \times \boldsymbol{v}$ con un tercer vector $\boldsymbol{w}$ para obtener un escalar $(\boldsymbol{u} \times \boldsymbol{v})$. $\boldsymbol{w}$. En vista de que $\boldsymbol{i} \cdot \boldsymbol{i}=\boldsymbol{j} \cdot \boldsymbol{j}=\boldsymbol{k} \cdot \boldsymbol{k}=\mathbf{1} ; \boldsymbol{i} \cdot \boldsymbol{j}=\boldsymbol{i} . \boldsymbol{k}=\boldsymbol{j} \cdot \boldsymbol{k}=\mathbf{0}$ si calculamos el producto mixto $(\boldsymbol{u} \times \boldsymbol{v})$. $\boldsymbol{w}$ se obtiene

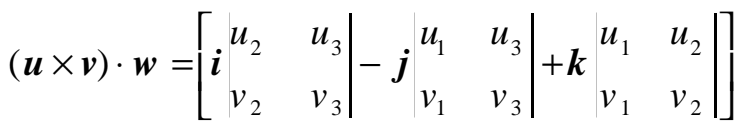

$$
\begin{aligned}
& \cdot\left(\boldsymbol{i} w_{1}+\boldsymbol{j} w_{2}+\boldsymbol{k} w_{3}\right) \\
& =w_{1} \begin{array}{ll}
u_{2} & u_{3} \\
v_{2} & v_{3}
\end{array}-w_{2}\left|\begin{array}{ll}
u_{1} & u_{3} \\
v_{1} & v_{3}
\end{array}\right|+w_{3} \mid \begin{array}{lll}
u_{1} & u_{2} \\
v_{1} & v_{2}
\end{array} \quad \text { (*) }
\end{aligned}
$$

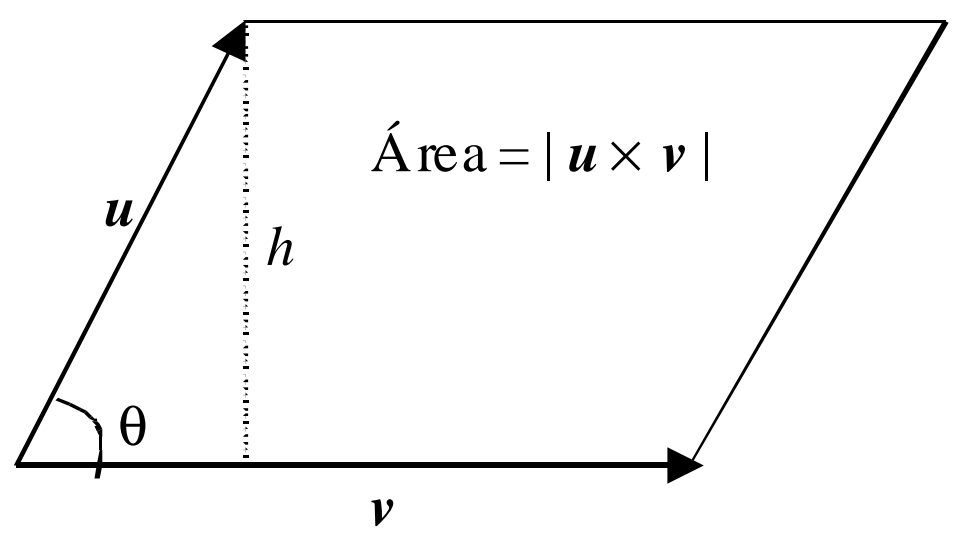

Figura 3 
La última expresión es el desarrollo por menores de la primera fila de un determinante cuyas filas son los componentes de los tres vectores, es decir,

$$
(\boldsymbol{u} \times \boldsymbol{v}) \cdot \boldsymbol{w}=\mid \begin{array}{lll}
u_{1} & u_{2} & u_{3} \\
v_{1} & v_{2} & v_{3} \\
w_{1} & w_{2} & w_{3}
\end{array}
$$

$=u_{1} v_{2} w_{3}+u_{2} v_{3} w_{1}+u_{3} v_{1} w_{2}-u_{3} v_{2} w_{1}-u_{1} v_{3} w_{2}-u_{2} v_{1} w_{3}$

de donde, por las propiedades de los determinantes, se ve claramente que los tres vectores juegan un papel simétrico en el producto mixto, por lo que es lo mismo escribir $(\boldsymbol{u} \times \boldsymbol{v})$. $\boldsymbol{w}$ que escribir $(\boldsymbol{w} \times \boldsymbol{u})$. $\boldsymbol{v} q u e$ escribir $\boldsymbol{u} .(\boldsymbol{v} \times \boldsymbol{w})$ donde hemos intercambiado la cruz y el punto, así como el cambio de posición de los paréntesis. Nótese que en vista de que la expresión $(\boldsymbol{u} . \boldsymbol{v}) \times \boldsymbol{w}$ no tiene sentido, puesto que se quiere hacer un producto cruz entre un escalar y un vector, operación que no está definida, algunos autores prescinden de los paréntesis y escriben para el producto mixto $(\boldsymbol{u} \times \boldsymbol{v})$. $\boldsymbol{w}=$ $[\boldsymbol{u}, \boldsymbol{v}, \boldsymbol{w}]=[\boldsymbol{w}, \boldsymbol{u}, \boldsymbol{v}]=[\boldsymbol{v}, \boldsymbol{w}, \boldsymbol{u}]$. Nótense las permutaciones cíclicas de los vectores entre corchetes. Se debe tener cuidado en no alterar el orden de los factores porque cada intercambio entre dos filas de un determinante cambia el signo del mismo (sin cambiar su valor absoluto). Una interpretación geométrica del producto mixto es que su valor absoluto es igual al volumen del paralelepípedo determinado por los tres vectores que intervienen como se ilustra en la figura 4. (Thomas, 1960).

El vector $(\boldsymbol{u} \times \boldsymbol{v})$ tiene como magnitud el área del paralelogramo determinado por los vectores $\boldsymbol{u}$ y $\boldsymbol{v}$. Dicho vector es ortogonal al plano del paralelogramo y al ser multiplicado con punto por el vector $\boldsymbol{w}$ el resultado es un escalar, cuyo valor es la distancia entre los paralelogramos marcados $\boldsymbol{A}$ y $\boldsymbol{B}$ en la figura 4, multiplicada por el área del paralelogramo $\boldsymbol{B}$ que está fungiendo como base de un prisma. El valor absoluto del resultado es igual al volumen del paralelepípedo.

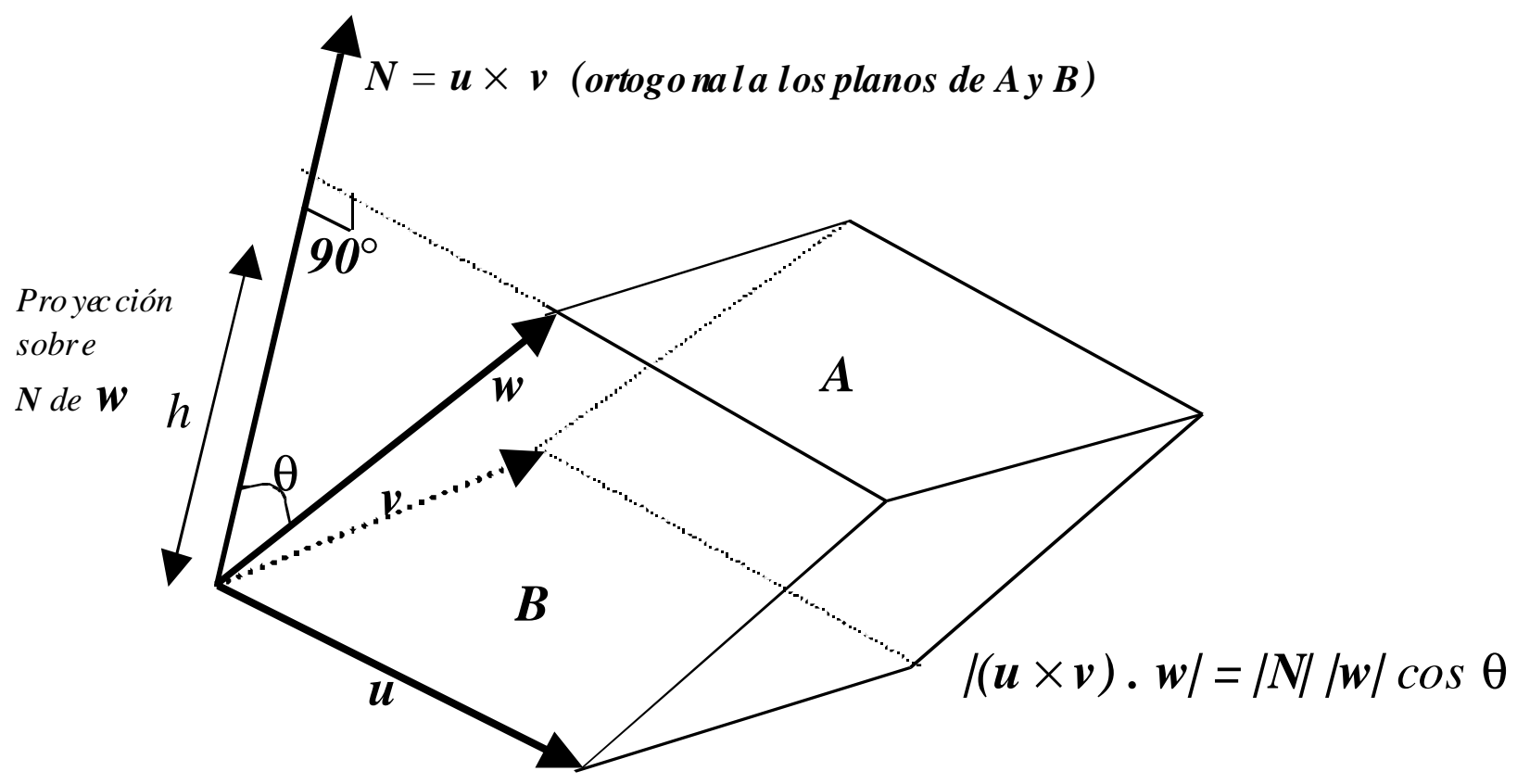

Figura 4 
En vista de la fórmula determinantal para el producto mixto, se tiene que el volúmen del paralelepípedo es igual al valor absoluto de un determinante

$$
V=\operatorname{abs}\left(\left|\begin{array}{lll}
u_{1} & u_{2} & u_{3} \\
v_{1} & v_{2} & v_{3} \\
w_{1} & w_{2} & w_{3}
\end{array}\right|\right)
$$

En un espacio de 4 dimensiones, cuatro vectores linealmente independientes determinan un paralelepípedo 4-dimensional. Su volumen se define como el valor absoluto de un determinante

$$
V=\operatorname{abs}\left[\begin{array}{llll}
t_{1} & t_{2} & t_{3} & t_{4} \\
u_{1} & u_{2} & u_{3} & u_{4} \\
v_{1} & v_{2} & v_{3} & v_{4} \\
w_{1} & w_{2} & w_{3} & w_{4}
\end{array}\right]
$$

Para paralelepípedos de $n$ dimensiones determinados por $n$ vectores linealmente independientes, el volumen se define por medio del valor absoluto de un determinante $n \times n$ en el que aparecen como filas las componentes de los vectores que determinan al paralelepípedo. En vista de que el determinante de una matriz es igual al determinante de su transpuesta, algunos autores ponen las componentes de los vectores que determinan un paralelepípedo en $n$ dimensiones como columnas de la matriz en vez de filas.

\section{Definición de un producto vectorial en $n$ dimensiones para $n>3$}

Una de las dificultades que se han encontrado para definir el producto vectorial de dos vectores en cuatro dimensiones ha sido la insistencia en considerar solamente dos vectores. El mismo problema se tiene para definir el producto vectorial de dos vectores en el plano (2 dimensiones). Si se usa la condición de que el producto sea ortogonal a los factores, en dos dimensiones no existe una dirección ortogonal a dos vectores no colineales. En cuatro dimensiones existe una infinidad (que genera una variedad lineal de dos dimensiones) de vectores ortogonales a los dos factores. Las dificultades desaparecen cuando uno se percata que la generalización del producto vectorial se vuelve muy natural cuando en vez de insistir en usar dos factores se utilizan $n-1$ factores para espacios $n$-dimensionales. La generalizaciónes directa por analogía, si en tres dimensiones el producto vectorial de $n-1=2$ vectores es

$$
\boldsymbol{u} \times \boldsymbol{v}=\left|\begin{array}{ccc}
u_{1} & u_{2} & u_{3} \\
v_{1} & v_{2} & v_{3} \\
\boldsymbol{i} & \boldsymbol{j} & \boldsymbol{k}
\end{array}\right|
$$

entonces en $n=4$ dimensiones la definición del producto de $n-1=3$ vectores es

$$
\boldsymbol{u} \times \boldsymbol{v} \times \boldsymbol{w}=\left|\begin{array}{cccc}
u_{1} & u_{2} & u_{3} & u_{4} \\
v_{1} & v_{2} & v_{3} & v_{4} \\
w_{1} & w_{2} & w_{3} & w_{4} \\
\boldsymbol{i} & \boldsymbol{j} & \boldsymbol{k} & \boldsymbol{I}
\end{array}\right|
$$

donde $\boldsymbol{i}, \boldsymbol{j}, \boldsymbol{k}, \boldsymbol{I}$ son cuatro vectores unitarios ortogonales entre sí que constituyen una base en el espacio de cuatro dimensiones, en términos de los cuales están dadas las cuatro componentes de los tres vectores.

Esta definición pro duce un vec tor tetradimensional, el cual tiene varias propiedades que le dan utilidad para ciertas aplicaciones: 
1. El vector resultante es ortogonal a los tres vectores fac tor $\boldsymbol{u}, \boldsymbol{v}, \boldsymbol{w}$ y por lo tanto, al hiperplano, en el cual yacen los tres vectores.

2. Si se hace el producto punto con un cuarto vec tor, el valor absoluto del resultado es el volumen del paralelepípedo tetradi mensional determinado por los cuatro vectores.

3. Una consecuencia del punto anterior es que se puede interpretar el producto vectorial de los tres vectores como un vector cuya magnitud es igual al hiperárea (o volumen) del paralelepípedo en tres dimensiones, determinado por los tres vectores $\boldsymbol{u}, \boldsymbol{v}, \boldsymbol{w}$.

4. El producto vectorial $\boldsymbol{u} \times \boldsymbol{v} \times \boldsymbol{w}$ es una función multilinear de las componentes de los factores y por lo tanto, por las propiedades de los determinantes, satisface la Ley Distributiva con respecto a la suma de escalares y la suma vectorial. Debido a esto se puede escribir el producto vec to rial $\boldsymbol{u} \times \boldsymbol{v} \times \boldsymbol{w}$ de la siguiente manera

$\left(\mathrm{u}_{1} \boldsymbol{i}+u_{2} \boldsymbol{j}+u_{3} \boldsymbol{k}+u_{4} \boldsymbol{l}\right) \times\left(v_{1} \boldsymbol{i}+v_{2} \boldsymbol{j}+v_{3} \boldsymbol{k}+v_{4} \boldsymbol{l}\right) \times$ $\left(w_{1} \boldsymbol{i}+w_{2} \boldsymbol{j}+w_{3} \boldsymbol{k}+w_{4} \boldsymbol{l}\right)$.

Al distribuir el producto vectorial sobre las sumas se obtienen tríos de vectores unitarios multiplicados por escalares. Los tríos se pueden reducir a un vec tor unitario aplicando la definición determinantal y el hecho de que los vectores unitarios tienen las componentes:

$\boldsymbol{i}=(1,0,0,0), \boldsymbol{j}=(0,1,0,0), \boldsymbol{k}=(0,0,1,0), \boldsymbol{l}=$ $(0,0,0,1)$

NOTA: Si los vectores $\boldsymbol{u}, \boldsymbol{v}, \boldsymbol{w}$, y el cuarto vector son linealmente dependientes, el paralelepípedo en cuatro dimensiones ya- cerá completamente en un hiperplano, plano o línea de menos de cuatro dimensiones y tendrá cero volumen. Si los vectores $\boldsymbol{u}, \boldsymbol{v}, \boldsymbol{w}$, son linealmente dependientes, el paralelepípedo en tres dimensiones que determinan yacerá completamente en un plano o una línea y tendrá cero volumen.

En ese caso, el vector resultante del producto cruz será el vector cero que se puede considerar ortogonal a cualquier vector.

Para demostrar que el vector resultante del producto vectorial $\boldsymbol{u} \times \boldsymbol{v} \times \boldsymbol{w}$ es ortogonal a sus tres factores, supongamos que $\boldsymbol{u}, \boldsymbol{v}, \boldsymbol{w}$ son linealmente independientes y consideremos el producto punto de cada uno de los vectores por el producto vec to rial $\boldsymbol{u} \times \boldsymbol{V} \times \boldsymbol{w}$. Si llamamos al determinante $\boldsymbol{D}$ (que es un vec tor debido a los vectores unitarios $\boldsymbol{i}, \boldsymbol{j}, \boldsymbol{k}, \boldsymbol{I})$ y éste se expande por menores de la última fila, llamando a dichos menores $D_{1}, D_{2}, D_{3}$, $D_{4}$, (que son escalares) se obtiene

$$
\boldsymbol{D}=(-1)^{n+1}\left(\boldsymbol{i} D_{1}-\boldsymbol{j} D_{2}+\boldsymbol{k} D_{3}-\boldsymbol{l} D_{4}\right)
$$

Si ahora se hace el producto punto de $\boldsymbol{D}$ con cualquiera de los vectores $\boldsymbol{u}, \boldsymbol{v}, \boldsymbol{w}$ se obtiene un determinante que es igual a $\boldsymbol{D}$, pero en el cual se sustituye la última fila por el vec tor $\boldsymbol{u}, \boldsymbol{v}$ o $\boldsymbol{w}$ según sea el caso. Como el determinante así formado tiene dos filas iguales, su valor es cero, lo cual establece que el producto punto del determinante $\boldsymbol{D}=\boldsymbol{u} \times \boldsymbol{V} \times \boldsymbol{w}$ porcualquiera de los tres vectores $\boldsymbol{u}, \boldsymbol{v}, \boldsymbol{w}$ es nulo y por tanto $\boldsymbol{u} \times \boldsymbol{v} \times \boldsymbol{w}$ es ortogonal simultáneamente a los tres vectores $\boldsymbol{u}, \boldsymbol{v}, \boldsymbol{w}$. El caso en que $\boldsymbol{u}, \boldsymbol{v}, \boldsymbol{w}$ no sean linealmente independientes está tratado en la NOTA an te rior. 
De manera análoga al desarrollo que se realizó antes para la ecuación ( ${ }^{*}$ ), el producto punto de un cuarto vector $\boldsymbol{z}$ por el producto vectorial $\boldsymbol{u} \times \boldsymbol{v} \times \boldsymbol{w}$ resulta en el determinante cuyas filas son los componentes de los cuatro vectores, los cuales determinan un paralelepípedo en cuatro dimensiones. El valor absoluto de dicho determinante es (por definición) igual al volúmen del paralelepípedo, por lo cual $\boldsymbol{u} \times \boldsymbol{v} \times \boldsymbol{w}$ es un vector cuya magnitud es igual al volúmen del paralelepípedo determinado por los tres vectores $\boldsymbol{u}, \boldsymbol{v}, \boldsymbol{w}$ (de forma análoga a cómo $\boldsymbol{u} \times \boldsymbol{v}$ es el área del paralelogramo determinado por los vectores $\boldsymbol{u}, \boldsymbol{v}$, y que al multiplicarlo con punto por un tercer vector $\boldsymbol{w}$, el valor absoluto del resultado es igual al volumen del paralelepípedo en 3 dimensiones, determinado por los vectores $\boldsymbol{u}, \boldsymbol{v}$, $\boldsymbol{w})$. Si los vectores $\boldsymbol{u}, \boldsymbol{v}, \boldsymbol{w}$ son linealmente dependientes, el producto $\boldsymbol{u} \times \boldsymbol{V} \times \boldsymbol{w}$ es el vec tor cero, ya que se estaría calculando el volumen de un paralelepípedo que yace totalmente en un plano bidimensional o en una línea. (Estamos excluyendo en todas las discusiones el caso trivial en que alguno de los tres factores $\boldsymbol{u}, \boldsymbol{v}, \boldsymbol{w}$ sean el vec tor cero).

La extensión de las anteriores definiciones para encontrar el producto cruz entre $n-1$ vectores en un espacio $n$ dimensional, es directa. Llamemos a los $n-1$ vectores $\boldsymbol{a}, \boldsymbol{b}, \ldots$ ., $\boldsymbol{d}$, cuyas componentes, usando como base vectores $\boldsymbol{i}, \boldsymbol{j}, \boldsymbol{k}$, . . . , $\boldsymbol{l}$, mutuamente ortogonales, unitarios, sobre y en el sentido de los $n$ ejes cartesianos, son respectivamente

$\left(a_{1}, a_{2}, \ldots, a_{n}\right),\left(b_{1}, b_{2}, \ldots, b_{n}\right), \ldots,\left(d_{1}, d_{2}, \ldots\right.$, $\left.d_{n}\right)$

entonces el producto cruz $\boldsymbol{a} \times \boldsymbol{b} \times \ldots \boldsymbol{d} \times$ está dado por

$$
\boldsymbol{a} \times \boldsymbol{b} \times . . \times \boldsymbol{d}=\begin{array}{ccccc}
a_{1} & a_{2} & a_{3} & \ldots & a_{n} \\
b_{1} & b_{2} & b_{3} & \ldots & b_{n} \\
\ldots & \ldots & \ldots & \ldots & \ldots \\
d_{1} & d_{2} & d_{3} & \ldots & d_{n} \\
\boldsymbol{i} & \boldsymbol{j} & \boldsymbol{k} & \ldots & \boldsymbol{l}
\end{array} \mid \begin{gathered}
\\
\downarrow-1 \\
\end{gathered}
$$

La definición dada tiene las mismas propiedades del producto cruz definido para 3 y 4 dimensiones. La demostración y discusión se extienden sin problema y las omitimos, ya que es muy parecida a la que aparece en la literatura para tres dimensiones (Kaplan, 1952); (Sokolnikoff, 1958); (Thomas, 1960) y (Hildebrand, 1962), que el producto vectorial de $n-1$ vectores en un espacio $n$ dimensional es distributiva con respecto a la suma escalar y la suma vec to rial. En cuanto a la ley conmutativa y la asociativa, ya nuestra experiencia en 3 dimensiones nos in dica que no se cumplen. A este respecto, es preferible considerar al producto vec to rial como una función de $n-1$ variables que están ordenadas. Las permutaciones de dichas vari ables le pueden cambiar el signo a la función.

\section{Ilustraciones numéricas de las aplicaciones del producto cruz entre $n-1$ vectores en un espacio $n$ dimensional}

Ante todo, conviene señalar que para el caso de 3 dimensiones el producto de $n-1$ vectores coincide totalmente con el producto cruz ordinario del álgebra vectorial que se utiliza en la física e ingeniería. Para 2 dimensiones se utilizaría un solo factor y el resultado del producto vectorial con un solo factor es un vector ortogonal a dicho factor. Si el factores un vector bidimensional que tiene coordenadas $x_{1}, y_{1}$ con respecto a un 
par de ejes coordenados cartesianos entonces el producto cruz de dicho vec tor es

$$
\begin{array}{rr}
x_{1} & y_{1} \\
\boldsymbol{i} & \boldsymbol{j}
\end{array}=-y_{1} \boldsymbol{i}+x_{1} \boldsymbol{j}
$$

el cual es claramente ortogonal al vector original (Figura 5) ya que el producto punto de los dos vectores es cero: $-x_{1} y_{1}+x_{1} y_{1}=0$. Si introducimos un segundo vector $\left(x_{2}, y_{2}\right)$ no colineal con $\left(x_{1}, y_{1}\right)$ los dos vectores $\left(x_{1}, y_{1}\right)$ $y\left(x_{2}, y_{2}\right)$ determinan un paralelogramo como se muestra en la figura 5. El "volumen" (área por el número de dimensiones) del paralelogramo, está dado por el valor absoluto del producto punto entre el producto cruz del primer vector y el segundo vector, es decir $|(-y 1, x 1) .(x 2, y 2)|=\left|x_{1} y_{2}-x_{2} y_{1}\right|$.

Dicho "volumen" también se puede calcular por medio del determinante

$$
\operatorname{abs}\left[\mid \begin{array}{ll}
x_{1} & y_{1} \\
x_{2} & y_{2}
\end{array}\right]=\left|x_{1} y_{2}-x_{2} y_{1}\right|
$$

logrando el mismo resultado. Los resultados se pueden verificar geométricamente restándole al área del rectángulo OEAD los trapecios y triángulos que no forman parte del paralelogramo OCAB de la figura 6 .

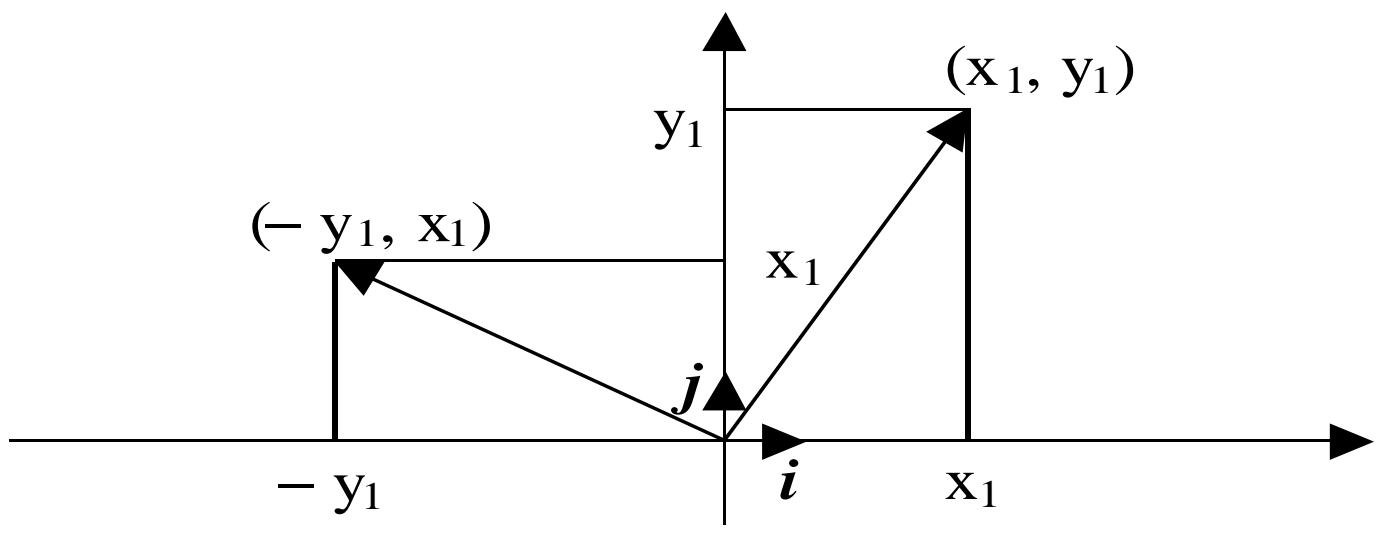

Figura 5

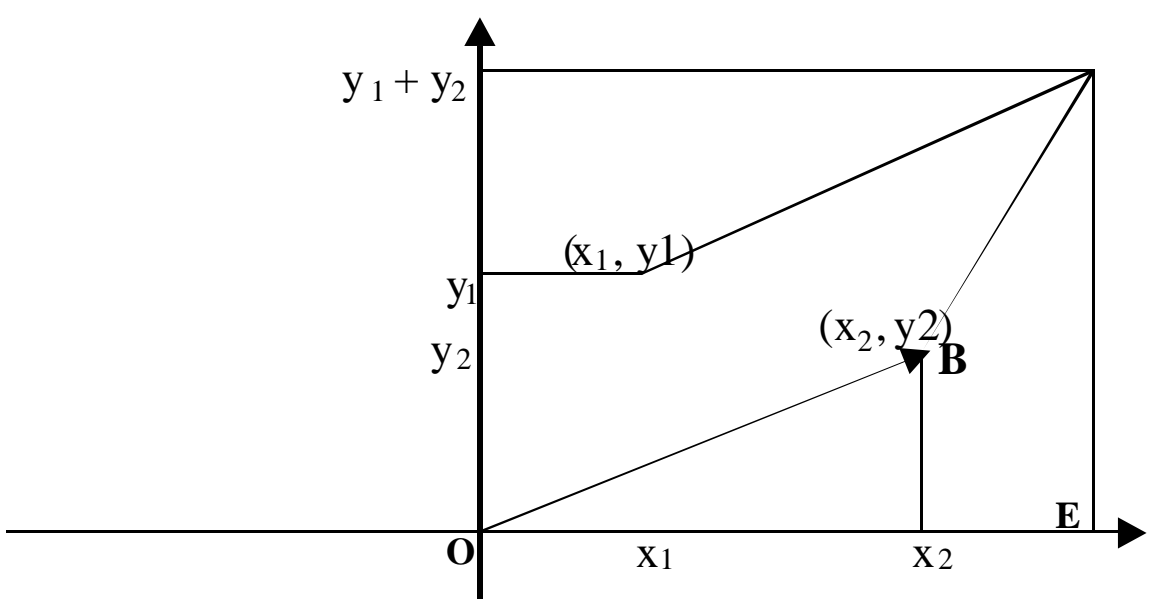

Figura 6 
Ya vimos que el producto cruz de $n-1$ vectores en un espacio $n$-di men sional es útil para calcular el volumen de un paralelepípedo de $n$ dimensiones, determinado por los $n-1$ vectores factor y un enésimo vector, así también que el resultado es un escalar dado por el producto mixto del producto vectorial de los primeros $n-1$ vec- tores por el enésimo vector y es igual a un determinante de orden $n$ cuyas filas (o columnas, ya que el determinante de la matriz transpuesta es igual) son las componentes de los vectores con respecto a una base ortonormal cualquiera. El producto vectorial entre los primeros $n-1$ vectores sirve también para calcular el hiperárea (en el caso de 3 dimensiones, el área) del hiperparalelogramo en $n-1$ dimensiones (en el caso de 3 dimensiones, el paralelogramo) determinado por los $n-1$ vectores. El hipeárea está dada como un vec tor $n$-di men sional cuya magnitud (o longitud) es la medida del hiperárea y cuya dirección es ortogonal al hiperplano en el que yace el hiperparalelogramo (en el caso tridimen sional, el plano y paralelogramo) de ter- minado por los primeros $n-1$ vectores. De lo anterior, es fácil pasar a resolver problemas de volúmenes de triángulos, tetraedros y sus extensiones a más dimensiones (simplejos), lo cual se ilustrará con un ejemplo numérico.

Consideremos un ejemplo numérico muy simple en cuatro dimensiones. Por su simplicidad nos ahorraremos trabajo numérico; para otros casos, los conceptos son igual de simples y sólo se complica el trabajo numérico.

Construiremos un simplejo de cuatro dimensiones apoyando nuestra construcción sobre un tetraedro trirrectángulo en tres dimensiones. Sea un tetraedro trirrectángulo formado por la esquina de un prisma recto rectángular cuyo vértice trirrectángulo está en el origen $\mathrm{O}$ de un sistema cartesiano y cuyo plano oblicuo opuesto al vértice en el origen es el triángulo $A B C$ que se muestra en la figura 7.

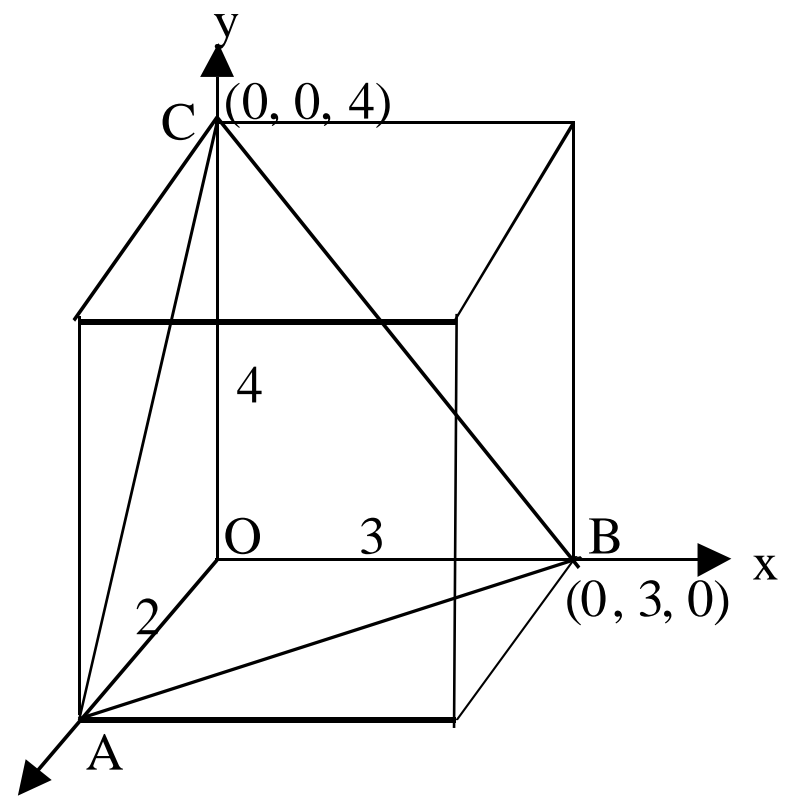

Figura 7 
Sobre cada eje hay un vector unitario (no se dibujan para no complicar la figura) y los vértices $A, B$ y $C$ son las puntas de las flechas (no dibujadas) de tres vectores cuyas componentes con respecto a la base dada por los vectores unitarios son los tríos de números junto a dichos vértices. En vista de que el tetraedro trirrectángulo $\mathrm{OABC}$ es un prisma triangular, podemos calcular su volumen usando la fórmula $V=b h / 3$, donde $b$ es el área de la base, para lo cual tomaremos el triángulo $\mathrm{OAB}$, aquí $h$ es la altura de la pirámide que en nuestro caso es 4. El área de la base vale $2 \times 3 / 2=3$. Por lo tanto, el volumen del tetraedro es $3 \times 4 / 3=4$.

Ahora incursionamos en la cuarta dirección, agregando un cuarto vector ortogonal a los otros 3 sobre un eje z (No dibujado en la figura 5). El cuarto vec tor tiene una longitud 4 y tiene su punta en el punto con coordenadas $(0,0,0,4)$. Los cuatro vectores tetradimensionales que generan el simplejo son entonces: $(2,0,0,0),(0,3,0,0)$, $(0,0,4,0)$ y $(0,0,0,4)$. Un simplejo en cuatro dimensiones tiene un hipervolumen de la cuarta parte del prisma con base tetraedral con igual altura (Xambó, 1997). Esto es una extensión del hecho de que un triángulo tiene la mitad del área del correspondiente paralelogramo generado por el mismo par de vectores bidimensional y un tetraedro tiene la tercera parte del volumen de un prisma tri angu lar generado por el mismo trío de vectores tridimensionales. Otra manera de calcular el hipervolumen de un simplejo en $n$ dimensiones es calcular el hiperárea de la base (que está en un hiperplano) y que está determinada por $n$ vértices del simplejo y multiplicarla por $(1 / n)$ de la distancia entre el vértice $n+1$ y el hiperplano de la base. Dicha distancia se mide sobre una línea ortogonal al hiperplano de la base. El hipervolumen del simplejo del ejemplo es igual al hiperárea del tetraedro que se usó como base (el tetraedro cuyo volumen calculamos anteriormente) multiplicado por una cuarta parte de la altura del simplejo con respecto a la base. Dicha altura medida sobre una línea perpendicular al hiperplano del tetraedro, (el hiperplano $\boldsymbol{w}-\boldsymbol{x}-\boldsymbol{y}$ ) que pase por el punto $D$ es la distancia sobre el eje $z$ entre $D$ y el origen, la cual vale 4 . El hipervolumen del simplejo es por lo tanto, $(4 \times 4) / 4=4$.

Revisemos nuestros cálculos usando productos cruz. Si estuviéramos calculando el volumen de un paralelepípedo en cuatro dimensiones, el volumen estaría dado por el valor del siguiente determinante

$$
\begin{array}{llll}
2 & 0 & 0 & 0 \\
0 & 3 & 0 & 0 \\
0 & 0 & 4 & 0 \\
0 & 0 & 0 & 4
\end{array}=96
$$

Como de lo que se trata es de un simplejo en 4 dimensiones, hay que dividir entre

$4 !=2 \times 3 \times 4=24$. (El dividendo 4! Proviene de que el triángulo introduce un factor de $1 / 2$, el tetraedro un fac tor de 1/3 y el simplejo un factor de $1 / 4$. En un simplejo de $n$ dimensiones, generalmente hay que usar un factor de $1 / n$ ! El volumen del simplejo es entonces $96 / 24=4$, que es el resultado obtenido anteriormente. Este resultado lo obtuvimos calculando

$$
(a \times b \times c) . d
$$

el cual es igual al determinante. Si deseamos obtener el hipeárea del tetraedro que se usó 
como base para el simplejo, entonces debemos calcular el producto cruz de los tres vectores que lo generan y multiplicarlo por $1 / 3$ !. Esto nos da

$$
(1 / 3 !) \mid \begin{array}{llll}
2 & 0 & 0 & 0 \\
0 & 3 & 0 & 0 \\
0 & 0 & 4 & 0 \\
\boldsymbol{i} & \boldsymbol{j} & \boldsymbol{k} & \boldsymbol{l}
\end{array}=4 \boldsymbol{u}
$$

El hiperárea del tetraedro es entonces $4 \mathrm{I}$. La magnitud 4 de este vec tor es el hiperárea del tetraedro, valor que coincide con el calculado por otros medios. El vector es ortogonal al hiperplano en el que está el tetraedro, el cual está generado por los vectores $\boldsymbol{i}, \boldsymbol{j}, \boldsymbol{k}$ y tiene como ecuación paramétrica $r_{1} \boldsymbol{i}+r_{2} \boldsymbol{j}+r_{3} \boldsymbol{k}$, donde $r_{1}, r_{2}$ y $r_{3}$ son números reales cualesquiera. $\mathrm{Al}$ multiplicar con punto esta expresión por el vector 4 I obtenemos el vec tor cero, toda vez que $\boldsymbol{i} \cdot \boldsymbol{I}=\boldsymbol{j} \cdot \boldsymbol{I}=\boldsymbol{k} \cdot \boldsymbol{I}=\mathbf{0}$ con lo que verificamos que el vector 4 I efectivamente es ortogonal al hiperplano en el cual está el tetraedro.

Otra de las aplicaciones del producto vecto rial de $n-1$ vectores en un espacio $n$-dimensional es a la solución de ecuaciones lineales simultáneas. Supóngase que se tiene la ecuación matricial

$$
A \boldsymbol{x}=\boldsymbol{b},
$$

la cual se puede escribir separando: las columnas $A_{1}, A_{2}, \ldots ., A_{n}$ de la matriz cuadrada $n \times n, A$, a la cual supondremos no singular, así como las componentes del vector $\mathbf{x}: \mathrm{x}_{1}, \mathrm{x}_{2}, \ldots, \mathrm{x}_{\mathrm{n}}$ como sigue:

$$
A_{1} x_{1}+A_{2} x_{2}+\ldots+A_{n} x_{n}=\boldsymbol{b}
$$

Supóngase que se puede encontrar un vec tor $H_{1}$ no nulo que sea ortogonal a las columnas $A_{2}, A_{3} \ldots, A_{n}$. En vista de que supusimos que la matriz $\boldsymbol{A}$ es no sin gu lar y que el vec tor es no nulo, el vector no puede ser al mismo tiempo ortogonal a la columna $A_{1}$. Si se multiplican ambos miembros de la última ecuación con punto por el vector $H_{1}$ mencionado se obtiene

$$
H_{1} . A_{1} x_{1}=H_{1} \cdot \boldsymbol{b}
$$

Como $H_{1}$. $A_{1}$ es un escalar diferente de cero, se puede pasar dividiendo al lado derecho obteniéndose

$$
x_{1}=H_{1} \cdot \boldsymbol{b} / H_{1} . A_{1}
$$

Repitiendo el proceso para cada una de las demás columnas, si llamamos $\mathrm{H}_{2}$ a un vec tor que es ortogonal a las columnas 1, 3, $4, \ldots, n ; H_{3}$ a uno que es ortogonal a las columnas $1,2,4, \ldots, n$; etc. y $H_{n}$ a uno que es ortogonal a las columnas $1,2,3, \ldots, n-1$ entonces tendremos

$$
\begin{gathered}
x_{2}=H_{2} \cdot \boldsymbol{b} / H_{2} \cdot A_{2} \\
\ldots \\
x_{n}=H_{n} \cdot \boldsymbol{b} / H_{n} \cdot A_{n}
\end{gathered}
$$

y hemos logrado resolver el sistema para cada una de las incógnitas. Solamente resta mostrar un método para calcular los vectores $H_{1}, H_{2} \ldots, H_{n}$.

El producto vectorial en $n$ dimensiones con $n-1$ factores nos proporciona un método de obtener un vec tor ortogonal a cada una de las columnas de la matriz $\boldsymbol{A}$ menos una. Tenemos entonces 
$H_{1}=$ producto vectorial de todas las columnas de $\boldsymbol{A}$ menos la primera.

$H_{2}=$ producto vectorial de todas las columnas de $\boldsymbol{A}$ menos la segunda.

$H_{n}=$ producto vectorial de todas las columnas de $\boldsymbol{A}$ menos la enésima.

Como ejemplo numérico consideremos el siguiente sistema

$$
\left(\begin{array}{cccc}
1 & 0 & 0 & -1 \\
0 & 1 & 2 & 0 \\
0 & -2 & 1 & 0 \\
0 & -1 & 0 & 3
\end{array}\right)\left(\begin{array}{l}
x_{1} \\
x_{2} \\
x_{3} \\
x_{4}
\end{array}\right)=\left(\begin{array}{c}
3 \\
0 \\
0 \\
-3
\end{array}\right)
$$

$\mathrm{Si}$ las columnas las escribimos como combinaciones lineales de los vectores unitarios ortonormales $\boldsymbol{i}, \boldsymbol{j}, \boldsymbol{k}, \boldsymbol{l}, \mathrm{y}$ hacemos el producto cruz de las columnas 2,3 , y 4 , obtenemos

$P_{234}=(\boldsymbol{j}-2 \boldsymbol{k}-\boldsymbol{l}) \times(2 \boldsymbol{j}+\boldsymbol{k}) \times(-\boldsymbol{i}+3 \boldsymbol{l})=$ $(\boldsymbol{j}-2 \boldsymbol{k}-\boldsymbol{l}) \times(-2 \boldsymbol{j} \boldsymbol{i}+6 \boldsymbol{j} \boldsymbol{l}-\boldsymbol{k} \boldsymbol{i}+3 \boldsymbol{k} \boldsymbol{l})$

en donde, en vista de la distributividad con respecto a la suma del producto cruz se ha calculado el producto de los dos últimos factores. Cuando en un producto aparecen dos vectores unitarios iguales se puede ignorar el término porque el producto es el vector cero. (Pues el determinante de la definición tiene dos filas iguales).

Ahora, haciendo el producto del resultado anterior con el primer fac tor (conservando cuidadosamente el orden ya que no hay conmutividad) e ignorando los productos con un fac tor repetido se obtiene

$P_{234}=-\boldsymbol{j} \boldsymbol{k} \boldsymbol{i}+3 \boldsymbol{j} \boldsymbol{k} \boldsymbol{l}+4 \boldsymbol{k} \boldsymbol{j} \boldsymbol{i}-12 \boldsymbol{k} \boldsymbol{j} \boldsymbol{l}+2 \boldsymbol{l} \boldsymbol{j} \boldsymbol{i}+$ $\boldsymbol{l} k \boldsymbol{i}$
Ahora, los productos de tres vectores unitarios son reemplazados por su resultado. Para deducir los resultados se pueden aplicar una variedad de reglas. Una de ellas es recordar que el producto de los $n-1$ primeros vectores da el último vec tor y luego ir haciendo permutaciones. Si la permutación es cíclica, en el caso que el número de dimensiones sea impar, no hay cambio de signo; en el caso de número de dimensiones par, en cada permutación cíclica se cambia el signo. Para $n=4$ se tendría haciendo permutaciones cíclicas de la permutación base: $\boldsymbol{i} \boldsymbol{j} \boldsymbol{k}=\boldsymbol{l}, \boldsymbol{j} \boldsymbol{k} \boldsymbol{l}=-\boldsymbol{i}, \boldsymbol{k} \boldsymbol{I} \boldsymbol{i}=\boldsymbol{j}, \boldsymbol{I} \boldsymbol{j} \boldsymbol{j}=-\boldsymbol{k}$. Las permutaciones entre dos símbolos adyacentes le cambian el signo al resultado. Por lo tanto: $\boldsymbol{j} \boldsymbol{i} \boldsymbol{k}=-\boldsymbol{l}, \boldsymbol{k} \boldsymbol{j} \boldsymbol{l}=\boldsymbol{i}, \boldsymbol{l} \boldsymbol{k} \boldsymbol{i}=-\boldsymbol{j}, \boldsymbol{i} \boldsymbol{l} \boldsymbol{j}=$ $k, i k j=-l, j l k=i, k i l=-j, l j i=k, j k i=l$, $k j i=-l, i k I=j$, etc.

Una segunda alternativa es aplicarle a los trios la definición de ter mi nan tal. Por ejemplo, para el trío $\boldsymbol{k} \boldsymbol{i} \boldsymbol{I}$ el determinante correspondiente es

$$
\left|\begin{array}{llll}
0 & 0 & 1 & 0 \\
1 & 0 & 0 & 0 \\
0 & 0 & 0 & 1 \\
\boldsymbol{i} & \boldsymbol{j} & \boldsymbol{k} & \boldsymbol{l}
\end{array}\right|=-\boldsymbol{j}
$$

El resultado se obtiene fácilmente desarrollando el determinante en menores de la columna en la que arriba del vector unitario de la cuarta fila aparecen solamente ceros (en nuestro caso la segunda columna). Hay que recordar la regla de signos del tablero de ajedrez para el desarrollo en menores (para nuestro caso, como el vector $\boldsymbol{j}$ está en la posición $(4,2)$ cuya suma es par, lleva signo "+" ) En nuestro caso, el menor del elemento $(4,2)$ da -1 . 
Usando los resultados anteriores se reemplazan los tríos por vectores unitarios de un solo símbolo y se obtiene

$P_{234}=-\boldsymbol{l}-3 \boldsymbol{i}-4 \boldsymbol{l}-12 \boldsymbol{i}-2 \boldsymbol{k}-\boldsymbol{j}=-15 \boldsymbol{i}-\boldsymbol{j}-\mathbf{2} \boldsymbol{k}$ $-5 l$

El resultado es un vector ortogonal a las tres últimas columnas de la matriz (El lector hará bien en verificarlo). Si multiplicamos el vector resultado con punto por la primera columna de la matriz y por el lado derecho obtenemos la siguiente ecuación escalar

(i). $(-15 i-j-2 k-5 l) x_{1}=(-15 i-j-2 k-5$ l). $(3 \boldsymbol{i}-3 \boldsymbol{l})$

$$
-15 x_{1}=-45+15
$$

de la que, despejando $x_{1}$ se obtiene

$$
x_{1}=2
$$

Procediendo en forma análoga, ya sin mostrar resultados intermedios, el producto vectorial de las columnas 1,3 y 4 da

$$
P_{134}=(\boldsymbol{i}) \times(2 \boldsymbol{j}+\boldsymbol{k}) \times(-\boldsymbol{i}+3 \boldsymbol{l})=3 \boldsymbol{j}-6 \boldsymbol{k}
$$

Al multiplicar con punto el vector resultante por la segunda columna y por el lado derecho, notamos que el producto con el lado derecho resulta cero, por lo que cualquier valor que de el producto con la segunda columna, con tal que sea diferente de cero, llevará a un valor de cero para $x_{2}$. Por lo tanto, $x_{2}=0$.

Procediendo en igual forma para las variables $x_{3}$ y $x_{4}$ se obtiene (dejamos los detalles al lector) $x_{3}=0, x_{4}=-1$.
Ahora demostraremos que el procedimiento funciona para cualquier matriz cuadrada $\boldsymbol{A}$ no sin gu lar. El producto vec to rial de todas las columnas menos la $i$-ésima de la matriz $n \times n$ $\boldsymbol{A}$ se puede escribir formalmente como el siguiente determinante

$$
H_{i}=\left|\begin{array}{cccc}
A_{11} & A_{21} & \ldots & A_{n 1} \\
\ldots & \ldots & \ldots & \ldots \\
A_{1, i-1} & A_{2 i-1} & \ldots & A_{n, i-1} \\
A_{1, i+1} & A_{2 i+1} & \ldots & A_{n, i+1} \\
\ldots & \ldots & \ldots & \ldots \\
A_{1 n} & A_{2 n} & \ldots & A_{n n} \\
\mathbf{i} & \mathbf{j} & \ldots & \mathbf{l}
\end{array}\right|
$$

Si se expande $H_{i}$ con respecto a los elementos de la última fila y posteriormente se hace el producto escalar con el vector $A_{i}$, en vista de que $\boldsymbol{i} \cdot \boldsymbol{i}=\boldsymbol{j} \cdot \boldsymbol{j}=\ldots .=\boldsymbol{I} . \boldsymbol{I}=1 \mathrm{el}$ resultado es la expansión del determinante igual a $H_{i}$, pero en el cual la última fila está formada por los componentes de la $i$-ésima fila. Este determinante se puede convertir en el determinante de la matriz $\boldsymbol{A}$ transpuesta, haciendo $n-i$ transposiciones, primero de la última fila con la penúltima, después de la penúltima con la antepenúltima, etc., hasta llegar a hacer la transposición de la i-ésima con la (i+1)-ésima. En vista de que cada transposición cambia el signo del determinante deducimos que

$$
H_{i} . A_{i}=(-1)^{n-i} \operatorname{det}\left(A^{T}\right), i=1,2, \ldots, n
$$

donde $\boldsymbol{A}^{\boldsymbol{T}}$ denota la matriz $\boldsymbol{A}$ transpuesta.

Por otra parte, el producto escalar de $H_{i}$ con $\boldsymbol{b}$ por las mismas razones que el caso recién analizado, es igual al determinante de $\boldsymbol{A}$ transpuesta al que le falta la résima fila y en la enésima fila aparecen las componentes del vector $\boldsymbol{b}$. Si a dicho determinante le 
hacemos las mismas transposiciones que las que le hicimos a $H_{i}$. $A_{i}$ se puede convertir en el determinante de $\boldsymbol{A}$ transpuesta, en el cual, en vez de la iésima fila aparece el vector $\boldsymbol{b}$ transpuesto. El factor $(-1)^{n-i}$ por el que hay que multiplicar a $H_{i} \cdot \boldsymbol{b}$, se cancela con el del denominador a la hora de hacer la división para calcular $x_{i}=H_{i} . \boldsymbol{b} / H_{i} . A_{i}$. Finalmente, en vista de que el determinante de una matriz cuadrada es igual al determinante de la matriz transpuesta, llegamos a la siguiente expresión para el cálculo de cada una de las incógnitas $x_{i}$

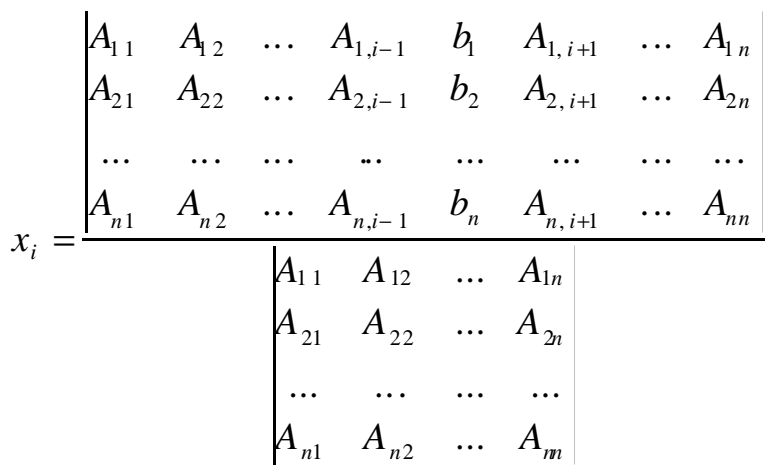

En esta expresión, en el denominador aparece el determinante de la matriz de coeficientes $\boldsymbol{A}$. En el numerador de la $i$-ésima vari able aparece el mismo determinante en el cual en lugar de la i-ésima columna de la matriz $\boldsymbol{A}$ aparece el vector $\boldsymbol{b}$ (lado derecho del sistema de ecuaciones). Esta última expresión es la conocida Regla de Cramer para resolver un sistema no singular de $n$ ecuaciones con $n$ incógnitas (Hohn, 1964).

\section{Conclusiones}

El producto cruz o producto vectorial de 2 vectores en tres dimensiones tiene suficientes particularidades para que se le haya dado el nombre de pseudovector o o vec tor axial. Una particularidad que llama la atención es que, por ejemplo, $i \times \boldsymbol{j}=\boldsymbol{k}$, teniendo $\boldsymbol{k}$ las mismas dimensiones lineales que $\boldsymbol{i}$ y $\boldsymbol{j}$ en vez de tener dimensiones de área (Hoffmann, 1966). Aunque en este artículo no se detalló el tema, en realidad se trata de un tensor antisimétrico de segundo orden que de casualidad tiene tres componentes independientes (además de las componentes que son nulas) que se transforman con cambios de referencias como un vector y que por esta razón en muchos sentidos se comporta como un vec tortridimensional. El mismo tensor en cuatro dimensiones tiene 6 componentes independientes en vez de 4 y por lo tanto, hay dificultades para obtener una definición útil del producto vectorial en cuatro y más dimensiones. En dos dimensiones el mismo tensor tiene una sola componente independiente, a la cual se le ha dado el nombre de pseudoescalar. Todo esto sucede cuando se considera el producto vectorial como una operación binaria y se busca una forma bilineal para calcularlo. Las dificultades desaparecen y se logra una definición útil si en vez de pensar en una operación binaria se considera al producto vectorial como una operación (n-1)-aria. Tomada esta decisión, la expresión determinantal del producto cruz de dos vectores en tres dimensiones se presta para hacer la extensión a $n$ dimensiones de una manera muy directa, utilizando las propiedades de los determinantes. Varias de las propiedades del producto vec to rial en tres dimensiones se conservan, entre ellas: 1) que el resultado de la operación es ortogonal a todos los factores; 2) que al hacer el producto punto con un vector adicional a los que participan en el producto vec to rial en $n$ dimensiones, la 
magnitud del resultado nos da el volumen del paralelepípedo determinado por los vectores que participaron en el producto vectorial y el vector adicional, dicho resultado tiene una expresión muy sencilla en términos de un determinante; 3) que si los factores de un producto vectorial son linealmente dependientes el producto es el vec tor cero, (en tres dimensiones el producto vectorial de dos vectores es el vector cero si los factores son colineales, el cual es un caso particular de dependencia lineal); 4) si los vectores participantes en el punto 2 son linealmente dependientes, el volumen del paralelepípedo es nulo y si son independientes el volumen es positivo (ya que está dado en términos del valor absoluto de un determinante).

Dada la íntima relación entre el producto vectorial y los determinantes y la de éstos con los sistemas de ecuaciones lineales simultáneas, seguramente el campo de las aplicaciones del producto vec to rial extendido a $n$ dimensiones resultará un campo muy fértil para interpretaciones y aplicaciones novedosas.

Algunos autores han atacado el problema del producto vec to rial en $n$ dimensiones con un número de factores arbitrarios por medio de funciones multilineares alternadas en forma abstracta (Lang, 1966). También Xambó (1997), proporciona un tratamiento geométrico menos general que Lang, también abstracto, con un grado mayor de abstracción que el dado en este artículo.

\section{Referencias}

Aleksandrov A.D., Kolmogorov A.N. y Lavrent'ev M.A. (1963). Mathematics: Its Content, Methods and Meaning. (3
Vols.). Cambridge, MA: The M. I. T. Press.

Birkhoff G. y Mac Lane S. (1965). A Survey of Modern Algebra. (Tercera Edición). Londres: Collier -Macmillan Limited.

Davis P.J. y Hersh R. (1981). The Mathematical Experience. Boston: Houghton Mifflin Company.

Dubrovin B, Nóvikov S. y Fomenko A. (1987). Geometría moderna: Métodos de la teoría de homologías. Editorial Mir, Moscú.

Hague B. (1951). An Introduction to Vector Analysis. Londres: Methuen and Company, Ltd.

Hildebrand F.B. (1967). Advanced Calculus for Applications. Englewood Cliffs, NJ: Prentice Hall, Inc.

Hoffmann B. (1966). About Vectors. Englewood Cliffs, NJ: Prentice Hall Inc.

Hohn F.E. (1964). Elementary Matrix Algebra. New York: The MacMillan Company.

Kaplan W. (1952). Advanced Calculus. Reading, MA: Addison-Wesley Publishing Company, Inc.

Lang S. (1966). Linear Algebra. Reading, MA: Addison-Wesley Publishing Company.

Smirnov V.I. (1970). Linear Algebra and Group Theory. New York: Dover Publi cations, Inc.

Sokolnikoff I.S. y Redheffer R.M. (1958). Mathematics of Physics and Modern Engineering. New York: Mc Graw Hill Book Company, Inc.

Thomas Jr. G.B. (1960). Calculus and Analytic Geometry. Reading, MA: Addison Wesley Publishing Company.

Xambó-Descamps S. (1997). Geometría. Barce lona: Edicions UPC. 
M.A. Murray-Lasso

\section{Semblanza del autor}

Marco Antonio Murray-Lasso. Realizó la licenciatura en ingeniería mecánica-eléctrica en la Facultad de Ingeniería de la UNAM. El Instituto de Tecnología de Massachussetts (MIT) le otorgó los grados de maestro en ciencias en ingeniería eléctrica y doctor en ciencias cibernéticas. En México, ha laborado como investigador en el Instituto de Ingeniería y como profesor en la Facultad de Ingeniería (UNAM) durante 43 años; en el extranjero, h a sido asesor de la NASA en diseño de circuitos por computadora para aplicaciones espaciales, investigador en los Laboratorios Bell, así como profesor de la Universidad Case Western Reserve y Newark College of Engineering, en los Estados Unidos. Fue el pres i dente fundador de la Academia Nacional de Ingeniería de México; vicepresidente y pres idente del Consejo de Academias de Ingeniería y Ciencias Tecnológicas (organización mundial con sede en Washington que agrupa las Academias Nacionales de Ingeniería) y secretario de la Academia Mexicana de Ciencias. Actualmente es jefe de la Unidad de Enseñanza Auxiliada por Computadora de la División de Estudios de Posgrado de la Facultad de Ingeniería de la UNAM, investigador nacional en ingeniería, consejero educativo del MIT y consultor de la UNESCO. 